\title{
ORIGINAL ARTICLE Phosphorylated neurofilament subunit NF-H as a biomarker for evaluating the severity of spinal cord injury patients, a pilot study
}

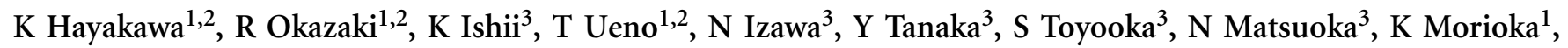

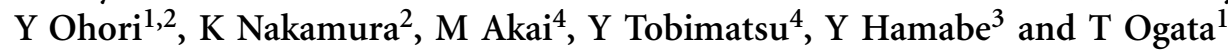

Study design: A pilot cross-sectional study of patients with acute cervical spinal cord injury (SCI).

Objectives: The precise evaluation of the severity of $\mathrm{SCl}$ is important for developing novel therapies. Although several biomarkers in cerebrospinal fluid have been tested, few analyses of blood samples have been reported. A novel biomarker for axonal injury, phosphorylated form of the high-molecular-weight neurofilament subunit NF-H (pNF-H), has been reported to be elevated in blood from rodent $\mathrm{SCl}$ model. The aim of this study is to investigate whether pNF-H values in blood can serve as a biomarker to evaluate the severity of patients with $\mathrm{SCl}$.

Setting: Tokyo Metropolitan Bokutoh Hospital and National Rehabilitation Center, Japan.

Methods: This study enrolled 14 patients with acute cervical SCl. Sequential plasma samples were obtained from $6 \mathrm{~h}$ to 21 days after injury. Patients were classified according to American Spinal Injury Association impairment scale (AIS) at the end of the follow-up (average, 229.1 days). Plasma pNF-H values were compared between different AIS grades.

Results: In patients with complete $\mathrm{SCl}$, pNF-H became detectable at $12 \mathrm{~h}$ after injury and remained elevated at 21 days after injury. There was a statistically significant difference between AIS A (complete paralysis) patients and AIS C (incomplete paralysis) patients. Conclusions: Plasma pNF-H was elevated in accordance with the severity of $\mathrm{SCl}$ and reflected a greater magnitude of axonal damage. Therefore, pNF-H is a potential biomarker to independently distinguish AIS A patients (complete SCI) from AIS C-E patients (incomplete $\mathrm{SCl}$ ). However, further studies are required to evaluate its utility in predicting prognosis of patients in the incomplete category.

Spinal Cord (2012) 50, 493-496; doi:10.1038/sc.2011.184; published online 24 January 2012

Keywords: spinal cord injury; biomarkers; neurofilament; ELISA; clinical trial

\section{INTRODUCTION}

The pathophysiology of spinal cord injury (SCI) comprises both the initial mechanical insult and secondary damage caused by inflammation, hypoxia and excitotoxicity. ${ }^{1,2}$ Attempts have been made to establish an efficient therapeutic protocol either to reduce the tissue damage by preventing secondary damage or to restore the neural tissue. It has been accepted that such intervention would be more efficient at an early time point after injury rather than during the chronic stage. ${ }^{3}$ Therefore, many pharmacological and tissue-engineering therapies are now designed to be applied within the first month after injury. ${ }^{4,5}$

One of the obstacles against the innovation of novel therapies for SCI is the difficulty of determining the baseline severity of the injury. During the early period after injury when novel therapies would be applied, unstable conditions of patients, including the phenomenon of spinal shock, prevent precise evaluation of neurological status. ${ }^{6}$ Therefore, a novel system that evaluates markers other than neurological symptoms would be expected to increase the accuracy of the initial evaluation of severity. One of the ways is to assess the amount of tissue damaged by traumatic insult. ${ }^{7}$ Because the central nervous tissue releases various structural proteins into the cerebral spinal fluid or peripheral blood flow upon trauma, attempts have been made to measure the concentration of a specific protein in samples of blood or cerebrospinal fluid after SCI. ${ }^{8-11}$ However, the utility of any proteins from blood samples as biomarkers for evaluating the severity of SCI patients has not yet been confirmed. A recent report by Shaw et al. ${ }^{12}$ introduced a novel biomarker, the phosphorylated form of the highmolecular-weight neurofilament subunit NF-H (pNF-H). As pNF-H is one of the structural proteins of axon fibers that are not detectable in blood of healthy animals, elevation of this protein in blood implies the breakdown of axons. Ueno et al. ${ }^{13}$ reported the correlation between plasma pNF-H level at 3 days after injury and hind-limb functions at 4 weeks after injury in the rat contusion model. Regarding human patients, pNF-H level in blood has been reported to be elevated in patients with subarachnoid hemorrhage, traumatic brain injury and amyotrophic lateral sclerosis. ${ }^{14-16}$ However, pNF-H has not been evaluated in a series of human patients with SCI.

In this report, we attempted to measure pNF-H in peripheral blood from patients with SCI, as a pilot study. Our primary aims were to

\footnotetext{
${ }^{1}$ Department of Rehabilitation for the Movement Functions, Research Institute, National Rehabilitation Center for Persons with Disabilities, Saitama, Japan; ${ }^{2}$ Department of Orthopaedic Surgery, Graduate School of Medicine, The University of Tokyo, Tokyo, Japan; ${ }^{3}$ Trauma and Critical Care Center, Tokyo Metropolitan Bokutoh Hospital, Tokyo, Japan and ${ }^{4}$ Rehabilitation Hospital, National Rehabilitation Center for Persons with Disabilities, Saitama, Japan

Correspondence: Dr T Ogata, Department of Rehabilitation for the Movement Functions, National Rehabilitation Center for Persons with Disabilities, Toru Ogata, 4-1 Namiki, Tokorozawa, Saitama, Japan.

E-mail: ogata-toru@rehab.go.jp

Received 30 June 2011; revised 14 November 2011; accepted 3 December 2011; published online 24 January 2012
} 
determine whether (1) pNF-H is detectable in blood samples of patients with SCI and (2) the pNF-H level reflects the severity of the injury.

\section{METHODS}

\section{Patients}

We performed a cross-sectional study at a trauma and critical care medical center, and a research institute after obtaining approval from both institutional ethical review boards. Patients with traumatic cervical SCI dating from October 2009 were included in this study. We performed initial physical examination within $12 \mathrm{~h}$ after injury and followed up to at least 30 days from admission. Patients whose radiographic examination confirmed intracranial hemorrhage were excluded.

\section{Samples}

Blood samples of $5 \mathrm{ml}$ were collected at $6,12,18,24,48,72$ and $96 \mathrm{~h}$, and 6,8 , 10,14 and 21 days after the injury. Some samples were not collected at several time points. The plasma samples were collected after centrifugal separation, then frozen and stored until assay.

\section{Clinical evaluation}

The severity of neurological impairment was graded according to the American Spinal Injury Association impairment scale (AIS) by trained medical doctors. We confirmed that sacral-sparing was absent in the patients classified as AIS A. After the initial evaluation, the assessment was performed 7 and 30 days after injury. After discharge from the hospitals, the symptoms of patients were followed at an outpatient clinic. In some cases, their symptoms were confirmed by telephone interview by the doctors.

\section{Assay of pNF-H}

The pNF-H assay was carried out with a commercially available enzyme-linked immunosorbent assay kit (Human Phosphorylated Neurofilament H ELISA; BioVendor, Modrice, Czech Republic). The frozen plasma samples were allowed to thaw and were then diluted threefold with a dilution buffer. The assay was performed according to the manufacture's protocol. The standard curve was constructed by plotting the mean absorbance of the standards against the known concentration of the standards on a logarithmic scale with the fourparameter algorithm. The concentration of the sample was calculated from the standard curve. Assays for which the pNF-H level exceeded the highest standard $\left(4000 \mathrm{pg} \mathrm{ml}^{-1}\right.$ ) were repeated with more diluted samples. The limit of detection (23.5 $\mathrm{pg} \mathrm{ml}^{-1}$ ) was calculated from the mean absorbance of the blank (dilution buffer only) plus three standard deviations of the absorbance of blank. We defined the actual limit of detection as $70 \mathrm{pg} \mathrm{ml}^{-1}$ because the sample was diluted threefold. All samples were tested in duplicate, and the average value of each sample was calculated.

\section{Statistical analysis}

Values of pNF-H below the limit of detection $\left(70 \mathrm{pg} \mathrm{m}^{-1}\right)$ were treated as 0 for statistical analysis. For comparison of plasma pNF-H values between AIS C patients and AIS D patients, repeated measure analysis of variance (ANOVA) was used. On the other hand, we used Mann-Whitney's U test to compare pNF-H values of AIS A patients and AIS C patients because variance of pNF-H value in AIS A patients was much larger than that in AIS C patients. We performed a two-sided test, and differences with a $P$-value of $<0.05$ were considered statistically significant.

\section{RESULTS}

pNF-H was detectable in blood samples of patients with SCI As shown in Table 1, the average age of the patients was 57. 9 (30-88) years. The follow-up period ranged from 43 to 556 days (average 229.1 days) after injury. At the final follow-up, four patients were classified as AIS A, zero as AIS B, five as AIS C, four as AIS D and one as AIS E. Among 14 cases in the current study, pNF-H was detected $\left(>70 \mathrm{pg} \mathrm{ml}^{-1}\right)$ in 11 cases within $96 \mathrm{~h}$ after injury. Two of pNF-H negative cases were AIS D and the other case was AIS C. The plasma pNF-H levels of four patients who were graded as AIS A at the final follow-up are shown in Figure 1. In those patients, pNF-H was detectable in plasma from $12 \mathrm{~h}$ after injury $\left(221-825 \mathrm{pg} \mathrm{ml}^{-1}\right.$ ), and it increased until $96 \mathrm{~h}$ after injury (1919-8023 $\mathrm{g} \mathrm{ml}^{-1}$ ). Thus, pNF-H is detectable in blood samples from patients with severe SCI and the pNF-H value shows increasing pattern in early period.

\section{Plasma pNF-H level was elevated in accordance with the severity of the injury}

As a biomarker for SCI, one of the expectations for $\mathrm{pNF}-\mathrm{H}$ is that its concentration should be useful for predicting the prognosis of each patient. Therefore, we investigated if there was a difference in $\mathrm{pNF}-\mathrm{H}$ levels depending on injury severity. Figure 2a shows average pNF-H values of each AIS grade at each time point from $6 \mathrm{~h}$ to $96 \mathrm{~h}$ after injury. There is a marked difference between AIS A patients (complete SCI) and AIS C-E patients (incomplete SCI), with statistical significance from $18 \mathrm{~h}$ to $96 \mathrm{~h}$ after injury between AIS A and AIS C (average pNF-H value at $96 \mathrm{~h}$ : AIS A patients, $5260 \mathrm{pg} \mathrm{ml}^{-1}$; AIS C patients, $449 \mathrm{pg} \mathrm{m}^{-1}$ ). In terms of predictive usage of $\mathrm{pNF}-\mathrm{H}$, we observed one particular case, Case 11 (AIS C), who was initially evaluated as AIS A on admission and then showed improvement to

Table 1 Patients

\begin{tabular}{|c|c|c|c|c|c|c|c|c|c|}
\hline Case & Age & Mechanism of injury & Level & $\begin{array}{l}\text { AIS at initial } \\
\text { examination }\end{array}$ & $\begin{array}{l}\text { AIS at final } \\
\text { examination }\end{array}$ & $\begin{array}{l}\text { Follow-up period } \\
\text { (day post injury) }\end{array}$ & Complications & $\begin{array}{c}\text { Steroid } \\
\text { treatment }\end{array}$ & $\begin{array}{c}\text { Decompression } \\
\text { surgery }\end{array}$ \\
\hline 1 & 69 & Fall from standing height & C6 & C & $\mathrm{C}$ & 199 & OPLL & No & No \\
\hline 2 & 39 & MVA & C5 & C & $\mathrm{D}$ & 556 & None & No & No \\
\hline 3 & 69 & Fall & C5 & D & $\mathrm{D}$ & 435 & None & Yes & No \\
\hline 4 & 56 & Fall down the stairs & C5 & $\mathrm{D}$ & $E$ & 282 & None & Yes & No \\
\hline 5 & 60 & Fall down the stairs & C6 & $A$ & $A$ & 60 & None & Yes & No \\
\hline 6 & 58 & Fall & C5 & $\mathrm{D}$ & $\mathrm{D}$ & 497 & Gastric ulcer & Yes & No \\
\hline 7 & 59 & MVA & C6 & D & $\mathrm{D}$ & 43 & DM & No & No \\
\hline 8 & 88 & MVA & C6 & $\mathrm{C}$ & $\mathrm{C}$ & 60 & DM, RF & No & No \\
\hline 9 & 30 & Fall & C6 & $A$ & $A$ & 60 & Spleen injury & No & No \\
\hline 10 & 56 & Fall from standing height & C5 & $\mathrm{B}$ & $\mathrm{C}$ & 52 & None & No & No \\
\hline 11 & 65 & MVA & C6 & $A$ & $\mathrm{C}$ & 167 & OPLL & Yes & Yes \\
\hline 12 & 68 & Fall down the stairs & C5 & $A$ & A & 330 & Concusssion & No & Yes \\
\hline 13 & 59 & Fall from standing height & C6 & B & $\mathrm{C}$ & 295 & Gastric ulcer & No & No \\
\hline 14 & 35 & Sport & $\mathrm{C} 5$ & $A$ & $A$ & 172 & None & Yes & Yes \\
\hline
\end{tabular}

Abbreviations: DM, diabetes mellitus; MVA, motor vehicle accident; OPLL, ossification of the posterior longitudinal ligament; RF, renal failure. 


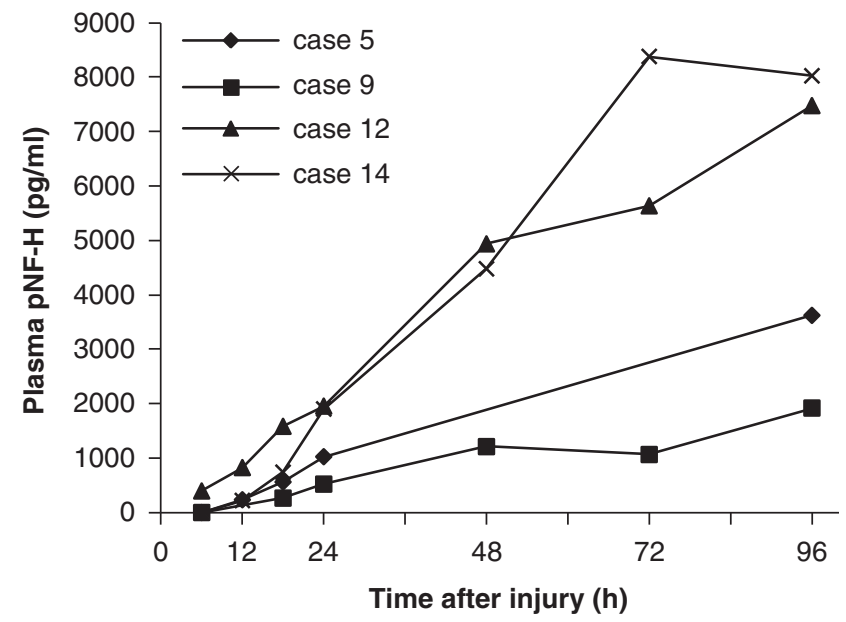

Figure 1 Time courses of plasma pNF-H values of 4 patients (Case 5, 9, 12 and 14) who were presented as AIS A at the final follow-up. The pNF-H was detectable in blood samples from $12 \mathrm{~h}$ after injury and showed an increase until $96 \mathrm{~h}$ after injury in all four cases.

become AIS C by 7 days after injury. In this 'eventual' AIS C case, we confirmed that the pNF-H value was much lower $\left(86 \mathrm{pg} \mathrm{ml}^{-1}\right.$ at $48 \mathrm{~h}$ and $689 \mathrm{pg} \mathrm{ml}^{-1}$ at $96 \mathrm{~h}$ after injury) than 'eventual' AIS A patients (Case 5, 9, 12 and 14: ranging from 1210 to $4935 \mathrm{pg} \mathrm{m}^{-1}$ at $48 \mathrm{~h}$ and from 1919 to $8023 \mathrm{pg} \mathrm{ml}^{-1}$ at $96 \mathrm{~h}$ after injury). As for less severe SCI, we observed that the pNF-H value tended to be higher in AIS C patients compared with AIS D patients $\left(264 \mathrm{pg} \mathrm{ml}^{-1}\right.$ in average at $96 \mathrm{~h}$ ), even if not statistically different (Figure 2b). It should be noted that the only one patient who was graded as AIS E presented relatively high pNF-H value (606 $\mathrm{pg} \mathrm{ml}^{-1}$ at $96 \mathrm{~h}$ ). His symptom was assessed as AIS D with central cord syndrome on admission, and then he recovered to be asymptomatic within 9 months.

Taken together, although we did not have any AIS B patients in our series, we assume that plasma pNF-H level is higher in AIS A patients (complete SCI) compared with AIS C-E patients (incomplete SCI), while the significance of pNF-H value in evaluating the severity of incomplete SCI remain undetermined.

Plasma pNF-H level remained elevated up to 21 days after injury In contrast to the rodent SCI model in which plasma pNF-H decreases after 3 days post injury, we found that pNF-H values in human SCI increase up to $96 \mathrm{~h}$ after injury. For a longer period of observation, we collected blood samples from four cases of AIS A and two cases of AIS C up to 21 days after injury. Figure 3 shows pNF-H values of each case, revealing that $\mathrm{pNF}-\mathrm{H}$ values remained elevated at 21 days after injury in all cases examined. Therefore, we assume that the kinetics of pNF-H are different between human and rodent SCI.

\section{DISCUSSION}

In the present pilot study, we analyzed blood samples from 14 patients with cervical SCI. Although the number of the patients and observational period were limited, we found that pNF-H was detectable in patients with SCI and that the pNF-H levels were significantly greater in AIS A patients (complete SCI) compared with AIS C-E patients (incomplete SCI). Therefore, we assume that $\mathrm{pNF}-\mathrm{H}$ can be regarded as a potential biomarker to assess the severity of SCI.
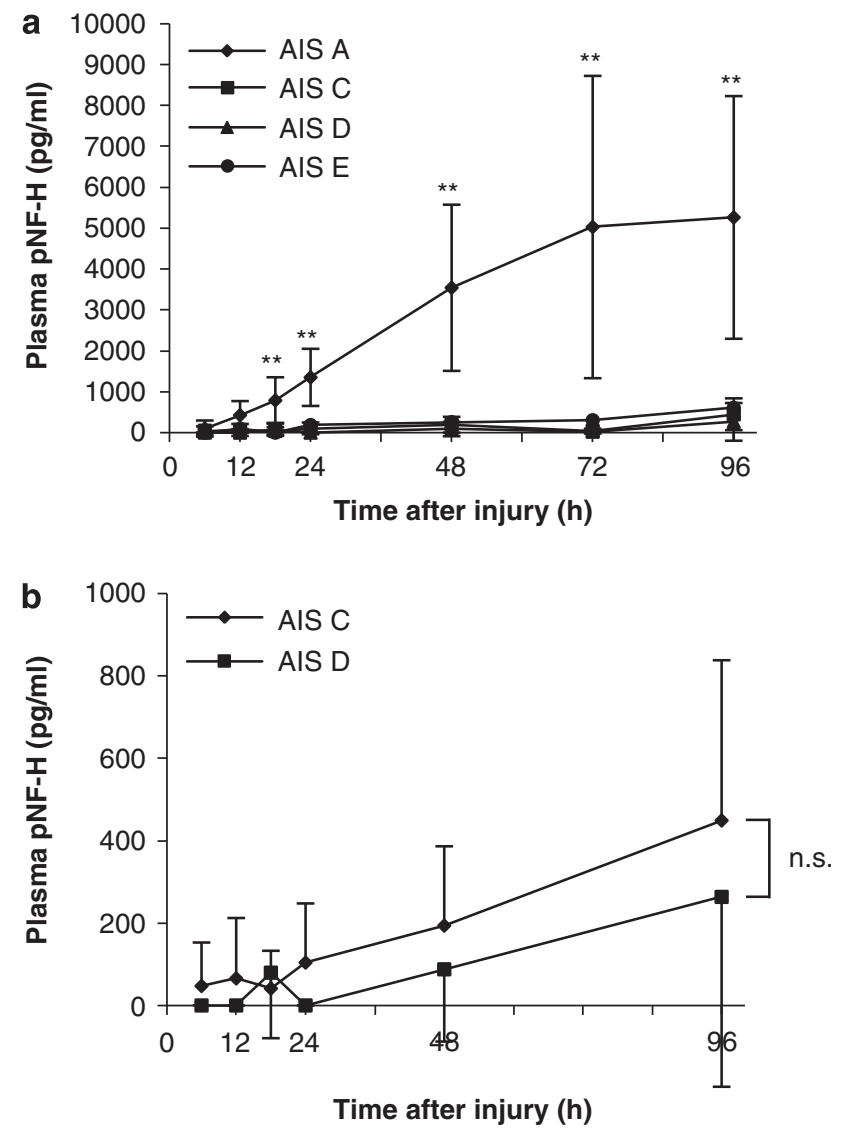

Figure 2 (a) Time courses of the average pNF-H values of each AIS grade $(A, C, D$ and $E)$ at each time point from $6 \mathrm{~h}$ to $96 \mathrm{~h}$ after injury. The difference between AIS A patients and AIS C patients was statistically significant from $18 \mathrm{~h}$ to $96 \mathrm{~h}$ after injury ${ }^{* *} P<0.01$, Mann-Whitney's $U$ test). (b) Time courses of the average pNF-H values of AIS grade $C$ and $D$ at each time point from $6 \mathrm{~h}$ to $96 \mathrm{~h}$ after injury. The pNF-H value tended to be higher in AIS C patients than in AIS D patients, though the difference was not statistically significant. Error bars indicate s.d.

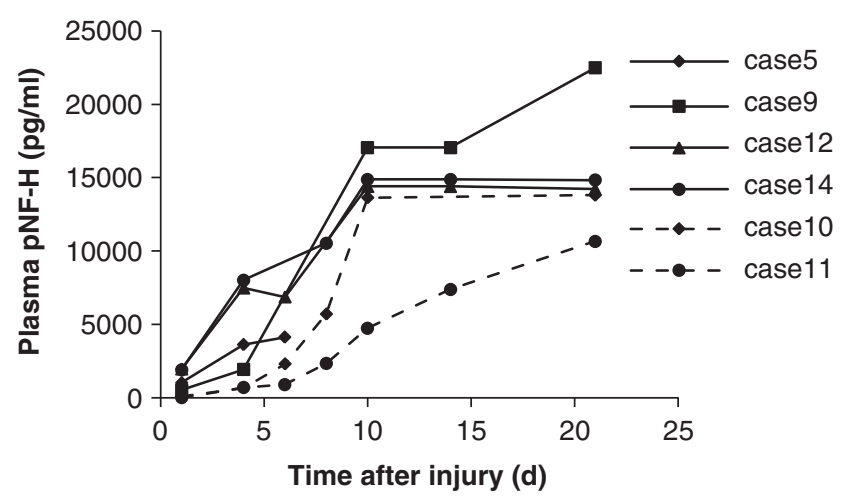

Figure 3 Time courses of plasma pNF-H values of 4 AIS A patients (Case 5, 9, 12 and 14; solid line) and 2 AIS C patients (Case 10 and 11; dashed line) until 21 days (for Case 5 until 6 days) after injury. Even at 21 days after injury, the pNF-H value remained elevated in all cases examined.

\section{pNF-H as a biomarker for central nervous system disorders}

The present study is the first report of plasma pNF-H measurement in a series of patients with SCI. The striking difference between the rat model and human patients is that the pNF-H values from patients 
remained elevated even at 21 days after injury, whereas the concentration of pNF-H in the rat has its peak at 3 days and returns to the baseline 14 days after injury. ${ }^{12}$ It remains unclear whether the difference is related to different patterns of axonal degeneration between humans and rats or to the difference in the condition of the blood-spinal cord barrier. Sustained elevation of pNF-H has also been reported in studies of subarachnoid hemorrhage and traumatic brain injury. ${ }^{15,16}$ The prolonged elevation of plasma pNF-H in patients with central nervous system disorders suggests the involvement of a continuous axonal degeneration, such as Wallerian degeneration or secondary axonal damage. In either case, it would be safe to conclude that the pNF-H level in blood represents the amount of axonal degeneration as a whole.

\section{Comparison to other biomarkers}

There have been several reports on the use of biomarkers to estimate prognosis among patients with SCI. For example, blood levels of S100B and NSE have been measured in experimental SCI. ${ }^{8,9,17}$ $\mathrm{S} 100 \mathrm{~B}$ is a calcium-binding protein localized predominantly in astrocytes and Schwann cells, and NSE is a glycolytic enzyme predominantly localized in the cytoplasm of neurons and cells of neuroendocrine lineage. ${ }^{9}$ Loy et al. ${ }^{8}$ reported that both $\mathrm{S} 100 \mathrm{~B}$ and NSE present monomodal patterns over time; S100B and NSE have their peak values at $6 \mathrm{~h}$ after injury and return to baseline at $24 \mathrm{~h}$. Such rapid changes require accurate timing of blood sampling, which is not always easy in the course of intensive care for SCI. Therefore, one of the favorable characteristics of pNF-H compared with those other markers is that the pNF-H can be stably detected at a later time point. On the other hand, pNF-H is barely detectable at $6 \mathrm{~h}$ after injury when S100B and NSE are already elevated in the blood. Taken together, these results suggest it could be beneficial to combine the use of S100B or NSE as 'early markers' and pNF-H as a 'delayed marker', depending on the type and timing of intervention applied.

\section{Limitations of the current study and future perspectives}

As the diagnosis is not definitive in the early stages after SCI because of the multiple traumas, sedation, spontaneous recovery etc., it is difficult to prove that a treatment that is administered during this period significantly improves neurological status. Therefore, the ideal biomarker is the one that enables the discrimination of, for example, definite AIS A patients from temporary AIS A patients, who would improve in neurological status with conventional therapies (as we observed in Case 11). Although the current study suggested that pNF-H has a potential for such biomarkers, further case-control studies are required to identify a possible threshold value to discriminate definite AIS A, and then to determine the predictive value of this biomarker. As for incomplete SCI, even though we observe the tendency of higher pNF-H values in AIS C compared with AIS D SCI, one case out of five cases of AIS C patients did not show an increase in pNF-H value. As there is a broad variation in limb functions among AIS C patients, we assume that further studies should include more detailed assessments for clinical symptoms, such as ASIA motor score, Spinal Cord Independence Measure (SCIM) and Walking Index for Spinal Cord Injury (WISCI) with a sufficient length of follow-up period.
Further studies are also required to obtain information about the influence of brain trauma, which may be a concomitant condition in SCI and may obscure the predictive ability of the biomarker. The data of the non-injured patients or patients without neuronal damages are also required to evaluate the specificity of this biomarker. In conclusion, we think the development of useful biomarkers for central nervous system trauma requires the accumulation of precise clinical follow-up data together with determination of the values of a variety of biomarkers in the acute phase.

\section{DATA ARCHIVING}

There were no data to deposit.

\section{CONFLICT OF INTEREST}

The authors declare no conflict of interest.

\section{ACKNOWLEDGEMENTS}

This study is funded by KAKENHI 22591649, a Grant-in-Aid for Scientific Research, Japan.

1 McDonald JW, Sadowsky C. Spinal-cord injury. Lancet 2002; 359: 417-425.

2 Crowe MJ, Bresnahan JC, Shuman SL, Masters JN, Beattie MS. Apoptosis and delayed degeneration after spinal cord injury in rats and monkeys. Nat Med 1997; 3: 73-76.

3 Houle JD, Tessler A. Repair of chronic spinal cord injury. Exp Neurol 2003; 182 : 247-260.

4 Kwon BK, Okon E, Hillyer J, Mann C, Baptiste D, Weaver LC et al. A systematic review of non-invasive pharmacologic neuroprotective treatments for acute spinal cord injury. J Neurotrauma 2011; 28: 1545-1588.

5 Tetzlaff W, Okon EB, Karimi-Abdolrezaee S, Hill CE, Sparling JS, Plemel JR et al. A systematic review of cellular transplantation therapies for spinal cord injury. J Neurotrauma 2011; 28: 1611-1682.

6 Bach-y-Rita P, Illis LS. Spinal shock: possible role of receptor plasticity and non synaptic transmission. Paraplegia 1993; 31: 82-87.

7 Kochanek PM, Berger RP, Bayir H, Wagner AK, Jenkins LW, Clark RS. Biomarkers of primary and evolving damage in traumatic and ischemic brain injury: diagnosis, prognosis, probing mechanisms, and therapeutic decision making. Curr Opin Crit Care 2008; 14: 135-141.

8 Loy DN, Sroufe AE, Pelt JL, Burke DA, Cao QL, Talbott JF et al. Serum biomarkers for experimental acute spinal cord injury: rapid elevation of neuron-specific enolase and S100beta. Neurosurgery 2005; 56: 391-397.

9 Cao F, Yang XF, Liu WG, Hu WW, Li G, Zheng XJ et al. Elevation of neuron-specific enolase and S-100beta protein level in experimental acute spinal cord injury. J Clin Neurosci 2008; 15: 541-544.

10 Pouw MH, Hosman AJ, van Middendorp JJ, Verbeek MM, Vos PE, van de Meent $\mathrm{H}$. Biomarkers in spinal cord injury. Spinal Cord 2009; 47: 519-525.

11 Kwon BK, Stammers AM, Belanger LM, Bernardo A, Chan D, Bishop CM et al. Cerebrospinal fluid inflammatory cytokines and biomarkers of injury severity in acute human spinal cord injury. J Neurotrauma 2010; 27: 669-682.

12 Shaw G, Yang C, Ellis R, Anderson K, Parker Mickle J, Scheff S et al. Hyperphosphorylated neurofilament NF-H is a serum biomarker of axonal injury. Biochem Biophys Res Commun 2005; 336: 1268-1277.

13 Ueno T, Ohori Y, Ito J, Hoshikawa S, Yamamoto S, Nakamura K et al. Hyperphosphorylated neurofilament NF-H as a biomarker of the efficacy of minocycline therapy for spinal cord injury. Spinal Cord 2011; 49: 333-336.

14 Boylan K, Yang C, Crook J, Overstreet K, Heckman M, Wang Y et al. Immunoreactivity of the phosphorylated axonal neurofilament $\mathrm{H}$ subunit (pNF-H) in blood of ALS model rodents and ALS patients: evaluation of blood pNF-H as a potential ALS biomarker. J Neurochem 2009; 111: 1182-1191.

15 Lewis SB, Wolper RA, Miralia L, Yang C, Shaw G. Detection of phosphorylated NF-H in the cerebrospinal fluid and blood of aneurysmal subarachnoid hemorrhage patients. J Cereb Blood Flow Metab 2008; 28: 1261-1271.

16 Zurek J, Bartlova L, Fedora M. Hyperphosphorylated neurofilament NF-H as a predictor of mortality after brain injury in children. Brain Inj 2011; 25: 221-226.

$17 \mathrm{Ma}$ J, Novikov LN, Karlsson K, Kellerth JO, Wiberg M. Plexus avulsion and spinal cord injury increase the serum concentration of S-100 protein: an experimental study in rats. Scand J Plast Reconstr Surg Hand Surg 2001; 35: 355-359. 